\title{
Scar sarcoidosis following blepharoplasty: a case report
}

\author{
Ji-Sang Min, MD, Eun-Jung Sohn, MD, Ji-Young Suh, MD, Hee-Bae Ahn, MD, PhD \\ Department of Ophthalmology, Dong-A University College of Medicine, Busan, Rep. of Korea
}

\begin{abstract}
Few cases have been previously reported regarding scar sarcoidosis following blepharoplasty. We report the case of a patient that developed sarcoidosis of a scar following a blepharoplasty procedure. A 41-year-old woman had a one-month history of beadshaped masses along her right upper eyelid. The patient underwent blepharoplasty 20 years ago. We performed excisional biopsy. Histopathological examination revealed a chronic non-caseating granuloma. Results of other tests were normal, including urinalysis, blood tests, and chest radiograph. Polymerase chain reaction (PCR) did not demonstrate evidence of tuberculosis, and a culture test for microbes showed no evidence of infection. She was diagnosed with sarcoidosis of the eyelid, and was prescribed an oral steroid. Once no evidence of recurrence was noted 3 months after the operation, the oral steroid dose was tapered off over an 8-week period. This case represents a rare occurrence of sarcoidosis that developed in the incisional scar after blepharoplasty. Sarcoidosis of the incisional scar should be considered as a differential diagnosis for an unusual mass that develops in a scar following blepharoplasty.
\end{abstract}

Keywords: biopsy; blepharoplasty; case report; excision; sarcoidosis; scar

\section{Background}

Sarcoidosis is a non-caseating granulomatous disease of unknown [1]. Twenty-five percent of sarcoidosis cases develop with ophthalmic lesions [1], and the most common ophthalmic related condition is anterior uveitis [2]. Sarcoidosis can develop in multiple organs, such as the lungs, lymph nodes, liver, spleen, phalangeal cartilage, eyes, and skin. Cutaneous sarcoidosis can develop in scars, although this is rare[3]. The causes of scar sarcoidosis include prior surgery [4], trauma [5], tattoos [6], a history of a herpes zoster infection, foreign tissues, and vaccinations. Nonetheless, there are no published reports of scar sarcoidosis developing after blepharoplasty. As such, it is difficult to consider scar sarcoidosis as a differential diagnosis for an unusual mass in a scar following blepharoplasty. We present a case of a patient with scar sarcoidosis who developed a beadlike appearing lesion after blepharoplasty.

\section{Materials and methods: case summary}

A 41-year old female patient presented with a 1-month history of bead-shaped masses on the right upper eyelid, despite a two-week course of oral steroid. A bilateral blepharoplasty was performed 20 years prior, with an insignificant history of trauma.

Erythema and tenderness to palpation were noted on physical examination. Diffuse, erythematous masses along the incision were noted on the ocular examination (Fig. 1A, B), and were confirmed by contrast computerized tomography (Fig. 1C). No specific findings were noted on slit-lamp microscopy.

Received December 28 2016, Revised March 23 2017, Accepted April 192017

Corresponding author: Hee-Bae Ahn, Department of Ophthalmology, Dong-A University College of Medicine, 26, Dongdaeshin-ro, Seo-gu, Busan 49201, Rep. of Korea

Tel: 82-51-240-5227, Fax: 82-51-254-1987, E-mail: hbahn@dau.ac.kr

This is an Open Access article distributed under the terms of the Creative Commons Attribution Non-Commercial License (http://creativecommons.org/licenses/by-nc/4.0), which permits unrestricted non-commercial use, distribution, and reproduction in any medium, provided the original work is properly cited.

Copyright $($ 2017. Korean Society of Korean Cosmetic Surgery and medicine (KSKCS \& KCCS). 

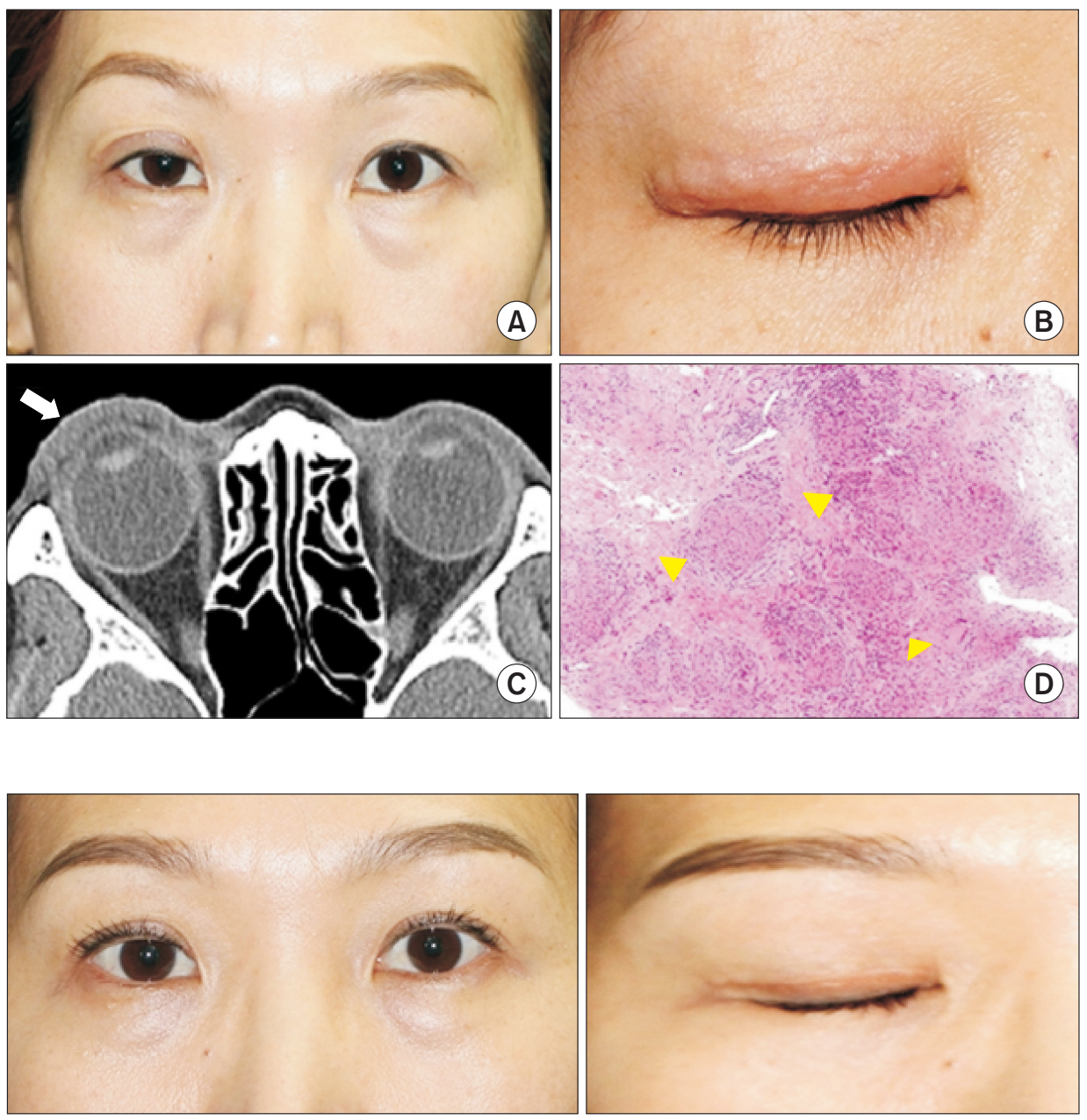

Fig. 1. A firm bead-shaped masses. (A,B) are visible along upper eyelid scar, and computed tomographic scan (C) show masses on upper eyelid. A photomicrograph of biopsy showing the numerous, noncaseating granulomas (arrow) with lymphocytes (D).

Fig. 2. Three month after treatment, there was dramatic resolution of the lesions of upper lid. And also there was no evidence of recurrence.
We prescribed $30 \mathrm{mg}$ of oral prednisolone to treat the inflammation reaction. Although the swelling started to subside, the size of the masses increased once the oral steroid dose was tapered to $10 \mathrm{mg}$; thus, we prepared for surgical intervention.

\section{Results}

Histopathologic examination revealed multiple noncaseating granulomas on H\&E staining, which was consistent with a diagnosis of scar sarcoidosis localized to the eyelid (Fig. 1D). Additional studies were performed to assess for systemic involvement. IgG subclass 4 level was within normal range, at $20.55 \mathrm{mg} / \mathrm{dl}$. All other relevant findings from laboratory studies and chest radiograph were normal. A polymerase chain reaction (PCR) test excluded tuberculosis, and no growth was noted on the microbial culture test.

The lesion recurred 1 month following the operation. We prescribed $40 \mathrm{mg}$ of oral steroid, tapered over an 8-week period. There was no evidence of recurrence at 3 months postop (Fig. 2).

\section{Discussion}

Sarcoidosis is a granulomatous inflammatory disease that can involve multiple organs. Additionally, 25\% of sarcoidosis patients develop cutaneous lesions and $25 \%$ are specific to the ophthalmic region. Most cases present with bilateral lymphadenopathy and restrictive lung disease. Several cases regarding eyelid scar sarcoidosis have been reported in the field of dermatology, but there is a paucity of literature regarding incisional sarcoidosis of the eyelid in the field of ophthalmology [7].

Incisional sarcoidosis following blepharoplasty is a rare occurrence. The patient in this case had blepharoplasty on her upper eyelids bilaterally, with masses along the incision isolated to her right eye. Sarcoidosis is often a diagnosis of exclusion. However, non-caseating granulomas on histopathologic evaluation increase suspicion, once other granuloma forming diseases have been excluded. In this case, a non-caseating granulomatous lesion was noted on $\mathrm{H} \& \mathrm{E}$ staining, and negative findings were noted for tuberculosis infection via PCR, as well as micro- 
bial cultures. Based on these findings, we diagnosed this patient with scar sarcoidosis.

The first line therapy for sarcoidosis localized on the eyelid involves the administration of oral steroids. The recurrence rate of sarcoidosis is only $7 \%$ after the administration of steroids with tapered dose [8]. When the patient does not respond to oral steroids, immunosuppressants such as methotrexate, can be used. In addition, local steroid injections can be effective [9]. In this case, we prescribed $30 \mathrm{mg}$ of an oral steroid, with dose tapered over 8 weeks. The recurring lesion led to us to perform an excisional biopsy. The patient was then administered $40 \mathrm{mg}$ of an oral steroid, which was gradually tapered over 8 weeks. No evidence of recurrence was noted 3 months after treatment.

\section{Conclusion}

Scar sarcoidosis following blepharoplasty is rare occurrencebut we believe that sarcoidosis should be considered as a differential diagnosis of masses that develop on blepharoplasty scars.

\section{Conflicts of interest}

The authors have nothing to disclose.

\section{References}

1. Newman LS, Rose CS, Maier LA. Sarcoidosis. N Engl J Med 1997;336:1224-34.

2. James DG. Ocular sarcoidosis. Ann N Y Acad Sci 1986;465:55163.

3. Kocak C, Yücel E, Namdar ND, Tak H. A case of scar sarcoidosis developing in an old scar area on the forehead. Acta Dermatovenerol Alp Pannonica Adriat 2015;24:61-3.

4. Tawfik HA, Assem M, Elkafrawy MH, Talib NM. Scar sarcoidosis developing 16 years after complete excision of an eyelid Basal carcinoma. Orbit 2008;27:438-40.

5. Kim YJ, Kim YD. A case of scar sarcoidosis of the eyelid. Korean J Ophthalmol 2006;20:238-40.

6. Murdoch SR, Fenton DA. Sarcoidosis presenting as nodules in both tattoos and scars. Clin Exp Dermatol 1997;22:254-5.

7. Kim H, Seol JE, Park IH, Kang JN, Sung HS, Jung SY. Scar sarcoidosis arising from various types of traumatized skin. Korean J Dermatol 2014;52:337-40.

8. Demirci H, Christianson MD. Orbital and adnexal involvement in sarcoidosis: analysis of clinical features and systemic disease in 30 cases. Am J Ophthalmol 2011;151:1074-80.el.

9. Prabhakaran VC, Saeed P, Esmaeli B, Sullivan TJ, McNab A, Davis G, et al. Orbital and adnexal sarcoidosis. Arch Ophalmol 2007;125:1657-62. 